\title{
Exciton and biexciton energies in bilayer systems
}

\author{
M. Y. J. Tan \\ St. Edmund's College, Mount Pleasant, Cambridge CB3 OBN, United Kingdom \\ N. D. Drummond and R. J. Needs \\ Theory of Condensed Matter Group, Cavendish Laboratory, University of Cambridge, Madingley Road, Cambridge, CB3 OHE, \\ United Kingdom \\ (Received 26 August 2004; published 4 January 2005)
}

\begin{abstract}
We report calculations of the energies of excitons and biexcitons in ideal two-dimensional bilayer systems within the effective-mass approximation with isotropic electron and hole masses. The exciton energies are obtained by a simple numerical integration technique, while the biexciton energies are obtained from diffusion quantum Monte Carlo calculations. The exciton binding energy decays as the inverse of the separation of the layers, while the binding energy of the biexciton with respect to dissociation into two separate excitons decays exponentially.
\end{abstract}

DOI: 10.1103/PhysRevB.71.033303

PACS number(s): 78.67.De, 02.70.Ss, 71.35.Cc

Bound excitons and biexcitons have been observed in semiconductors under a variety of conditions. In this paper we consider bilayer systems in which the electrons are spatially separated from the holes, leading to what are known as "indirect excitons." Such systems have been realized in double-quantum-well structures under an applied perpendicular electric field, which serves to confine electrons in one well and holes in the other. ${ }^{1,2}$ The possibility of BoseEinstein condensation of excitons in such structures has recently aroused much interest ${ }^{1-4}$ and there is a need for a deeper understanding of the processes that laser excitation initiates in these systems. In this paper we consider an aspect of excitations in coupled quantum wells which may be relevant to experiments on such systems-the energetics of biexcitons in bilayer systems.

The effective-mass approximation with isotropic electron and hole masses gives a simple description of excitons and biexcitons that has been applied to many systems. This model is highly idealized, and effects due to anisotropic masses, nonparabolic bands, and finite well widths and depths will be significant. The model is, however, simple enough to be solved to very high accuracy, providing benchmark results while still permitting comparisons with experimental data. We have calculated exciton and biexciton energies within the effective-mass approximation for a system consisting of ideal two-dimensional electron and hole layers separated by a distance $d$.

An exciton in an ideal two-dimensional bilayer geometry is described by the Schrödinger equation

$$
\begin{aligned}
& {\left[-\frac{\hbar^{2}}{2 m_{e}} \nabla_{e}^{2}-\frac{\hbar^{2}}{2 m_{h}} \nabla_{h}^{2}+\frac{e^{2}}{4 \pi \epsilon_{0}} \frac{1}{\sqrt{\left|\mathbf{r}_{e}-\mathbf{r}_{h}\right|^{2}+d^{2}}}\right] \Phi\left(\mathbf{r}_{e}, \mathbf{r}_{h}\right)} \\
& \quad=E \Phi\left(\mathbf{r}_{e}, \mathbf{r}_{h}\right),
\end{aligned}
$$

where $m_{e}$ and $m_{h}$ are the electron and hole masses, respectively, and $\epsilon$ is the static dielectric constant of the material. In the following, energies are given in terms of the exciton Rydberg, $\mathcal{R}^{*}=\mu_{e h} e^{4} /\left[2\left(4 \pi \epsilon_{0} \epsilon\right)^{2} \hbar^{2}\right]$, and lengths are given in terms of the exciton Bohr radius, $a_{B}^{*}=4 \pi \epsilon_{0} \epsilon \hbar^{2} /\left(\mu_{e h} e^{2}\right)$, where $\mu_{e h}=m_{e} m_{h} /\left(m_{e}+m_{h}\right)$ is the reduced mass.

Equation (1) may be simplified by transforming into the center-of-mass frame and separating the variables in cylindrical polar coordinates. For the zero-angular-momentum states we obtain

$$
-\frac{1}{r} \frac{\partial}{\partial r}\left(r \frac{\partial \Phi}{\partial r}\right)-\frac{2}{\sqrt{r^{2}+d^{2}}} \Phi=E_{X} \Phi,
$$

where $r$ is the in-plane component of the electron-hole separation. Equation (2) may be solved analytically when $d=0$, which gives a ground-state wave function of $\Phi(r) \propto \exp [$ $-2 r]$ and an energy of $E_{X}=-4 \mathcal{R}^{*}$. For $d>0$ we solved Eq. (2) using a standard Runge-Kutta numerical integration technique. The exciton energy is plotted as a function of $d$ in Fig. 1. The energy takes its minimum value of $-4 \mathcal{R}^{*}$ at $d=0$, while at small separations the energy varies linearly with $d$ and at large separations it varies as $1 / d$. The results shown in Fig. 1 may be fitted to the expression

$$
E_{X}=-\frac{4+A d+B d^{2}+C d^{3}}{1+D d+E d^{2}+F d^{3}+G d^{4}},
$$

where $A=154.363, B=648.9, \quad C=225.005, \quad D=46.4263$, $E=384.976, F=628.158$, and $G=129.672$. This expression

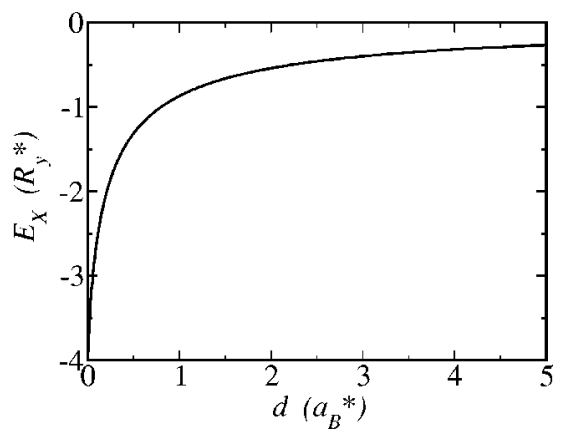

FIG. 1. Exciton energy as a function of the separation of the electron and hole layers. 
gives a maximum error of less than $0.0028 \mathcal{R}^{*}$ in the range $0<d<10 a_{B}^{*}$.

The Schrödinger equation for the biexciton is

$$
\begin{aligned}
& {\left[-\frac{1}{1+\sigma}\left(\nabla_{1}^{2}+\nabla_{2}^{2}\right)-\frac{\sigma}{1+\sigma}\left(\nabla_{a}^{2}+\nabla_{b}^{2}\right)+\frac{2}{r_{12}}+\frac{2}{r_{a b}}-\frac{2}{r_{1 a}}-\frac{2}{r_{1 b}}\right.} \\
& \left.-\frac{2}{r_{2 a}}-\frac{2}{r_{2 b}}\right] \Psi=E_{X X} \Psi
\end{aligned}
$$

where 1 and 2 denote the electron coordinates, $a$ and $b$ denote the hole coordinates, $r_{12}=\left|\mathbf{r}_{1}-\mathbf{r}_{2}\right|, r_{1 a}=\sqrt{\left|\mathbf{r}_{1}-\mathbf{r}_{a}\right|^{2}+d^{2}}$, etc., and $\sigma=m_{e} / m_{h}$. When expressed in units of $\mathcal{R}^{*}, E_{X}$ is a function only of $d$, but the biexciton energy, $E_{X X}$, is a function of both $d$ and $\sigma$. Equation (4) does not separate in cylindrical polar coordinates and we have to solve the manybody problem. For this purpose we have used the diffusion quantum Monte Carlo (DMC) method, which is a stochastic projector technique for solving the imaginary-time manybody Schrödinger equation. ${ }^{5}$ In the ground state of the biexciton the electrons have opposite spins and the holes have opposite spins, so the spatial part of the wave function is nodeless. The DMC method is exact in principle for nodeless wave functions, and although there are biases due to the use of finite time steps and populations of walkers, these can be made negligible for small systems such as this.

The sampling within DMC is guided by an approximate wave function that must be sufficiently accurate to give low statistical noise and to keep the biases small. The form of our approximate wave function was guided by the symmetries of the problem and the long- and short-distance behavior. The system composed of two separated bound excitons is always more stable than one consisting of four unbound charges. Therefore we expect the wave function to be exponentially small when all four particles are far apart. When one of the particles is far from the other three we expect the wave function to be exponentially small because the single charge will be attracted to the other three. Likewise we expect that the part of the wave function corresponding to one bound exciton and a free electron and hole is exponentially small. When $d$ is large we expect the system to consist essentially of two separated excitons, and the form of the approximate wave function must allow for this possibility. The short-range behavior of the wave function is fixed by the Kato cusp conditions, ${ }^{6}$ which ensure that the divergences in the potential and kinetic energies cancel when two particles are coincident. The biexciton wave function, $\Psi$, should be unaltered by exchange of (i) the two electron coordinates, or (ii) the two hole coordinates, i.e., $\Psi\left(\mathbf{r}_{1}, \mathbf{r}_{2}, \mathbf{r}_{a}, \mathbf{r}_{b}\right)=\Psi\left(\mathbf{r}_{2}, \mathbf{r}_{1}, \mathbf{r}_{a}, \mathbf{r}_{b}\right)$ $=\Psi\left(\mathbf{r}_{1}, \mathbf{r}_{2}, \mathbf{r}_{b}, \mathbf{r}_{a}\right)$, and when the electron and hole masses are equal, $\Psi$ should have the additional electron-hole symmetry $\Psi\left(\mathbf{r}_{1}, \mathbf{r}_{2}, \mathbf{r}_{a}, \mathbf{r}_{b}\right)=\Psi\left(\mathbf{r}_{a}, \mathbf{r}_{b}, \mathbf{r}_{1}, \mathbf{r}_{2}\right)$.

The binding between the excitons is expected to be small compared with the binding within an exciton. We therefore write the wave function as an appropriately symmetrized product of two exciton wave functions, which is then multiplied by a Jastrow function containing electron-electron and hole-hole terms. We use the following form, which satisfies all of the above conditions,

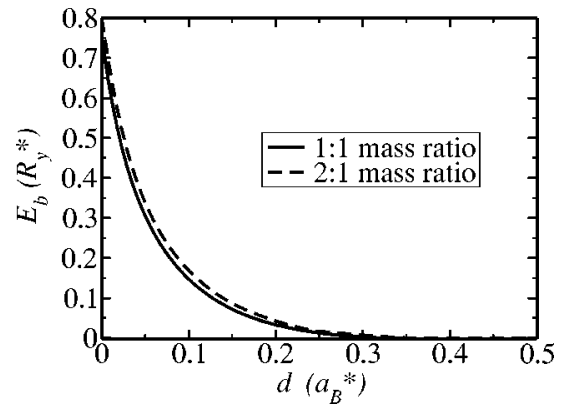

FIG. 2. The binding energy of the biexciton, $E_{b}=2 E_{X}-E_{X X}$, as a function of the separation of the electron and hole layers, $d$, for $\sigma=1$ and 2 . The error bars are smaller than the thickness of the lines.

$$
\begin{gathered}
\Psi=\Psi_{e e} \Psi_{h h} \Psi_{e h}, \\
\Psi_{e e}=\exp \left[\frac{c_{1} r_{12}}{1+c_{2} r_{12}}\right], \\
\Psi_{h h}=\exp \left[\frac{c_{3} r_{a b}}{1+c_{4} r_{a b}}\right], \\
\Psi_{e h}=\exp \left[\left(\frac{c_{5} r_{1 a}+c_{6} r_{1 a}^{2}}{1+c_{7} r_{1 a}}+\frac{c_{5} r_{1 b}+c_{8} r_{1 b}^{2}}{1+c_{9} r_{1 b}}+\frac{c_{5} r_{2 a}+c_{8} r_{2 a}^{2}}{1+c_{9} r_{2 a}}\right.\right. \\
\left.\left.+\frac{c_{5} r_{2 b}+c_{6} r_{2 b}^{2}}{1+c_{7} r_{2 b}}\right)\right] \\
+\exp \left[\left(\frac{c_{5} r_{1 a}+c_{8} r_{1 a}^{2}}{1+c_{9} r_{1 a}}+\frac{c_{5} r_{1 b}+c_{6} r_{1 b}^{2}}{1+c_{7} r_{1 b}}+\frac{c_{5} r_{2 a}+c_{6} r_{2 a}^{2}}{1+c_{7} r_{2 a}}\right.\right. \\
\left.\left.+\frac{c_{5} r_{2 b}+c_{8} r_{2 b}^{2}}{1+c_{9} r_{2 b}}\right)\right],
\end{gathered}
$$

where $c_{1}-c_{9}$ are parameters. This form is similar to that used by Lee et $a .^{7}$ for the three-dimensional biexciton, although our form has more flexibility in the electron-hole part. We require $c_{2}, c_{4}, c_{7}, c_{9}>0$ so that $\Psi$ is well behaved, and $c_{6}$, $c_{8}<0$ so that $\Psi_{e h}$ decays when the electrons and holes are far apart. Equation (5) describes two separated excitons when either $c_{6}$ or $c_{8}$ go to zero.

The values of the parameters $c_{1}$ and $c_{3}$ were fixed by the electron-electron and hole-hole Kato cusp conditions. ${ }^{6}$ The value of $c_{5}$ was fixed by the electron-hole cusp condition when $d=0$, while for $d>0$ there should be no electron-hole cusp and so we set $c_{5}=0$. When $\sigma=1$ electron-hole symmetry requires that $c_{1}=c_{3}$ and $c_{2}=c_{4}$. The optimal values of the remaining variable parameters were obtained by minimizing the variance of the variational energy. ${ }^{8,9}$

We calculated the energy of the biexciton, $E_{X X}$, as a function of $d$ for $\sigma=1$ and 2. Tests indicated that the time step and population control errors in the DMC results were negligible. All the Quantum Monte Carlo calculations were performed using the CASINO code. ${ }^{10}$ The biexciton binding energy with respect to dissociation into two separate excitons, $E_{b}=2 E_{X}-E_{X X}$, is plotted as a function of $d$ in Fig. 2 for $\sigma=1$ and 2. For small $d$ and $\sigma=1, E_{b}$ is close to the value 
obtained in earlier calculations for $d=0$ of $0.771 \mathcal{R}^{*} \cdot{ }^{11} E_{b}$ goes to zero at large $d$ much more rapidly than $E_{X}$ itself because the electron-electron and hole-hole repulsions dominate the electron-hole attraction in the biexciton at large $d$, tending to unbind the biexciton. Examination of the biexciton wave function shows that two separated excitons are formed at large $d$. The behavior of $E_{b}$ at large $d$ is reasonably well represented by a simple exponential form,

$$
E_{b}=\alpha \exp [-\beta d],
$$

where $\alpha=0.67573$ and $\beta=15.023$ for $\sigma=1$, and $\alpha=0.71714$ and $\beta=14.187$ for $\sigma=2$.

As a simple example of the use of these results we estimate the biexciton binding energy, $E_{b}$, in the experiments of Butov et al., ${ }^{1}$ who studied a system of two $80-\AA$-wide GaAs quantum wells separated by a 40 - $\AA$-wide barrier of $\mathrm{Al}_{0.33} \mathrm{Ga}_{0.67} \mathrm{As}$. The electron mass in GaAs is $m_{e}=0.067 m_{0}$, while the heavy-hole mass should be reduced from its bulk value of $0.45 m_{0}$ by confinement effects, and for simplicity we take a value of $m_{h}=0.134 m_{0}$. This gives a 2:1 mass ratio, although the results are not sensitive to the precise value of $m_{h}$. The value of $m_{h}=0.134 m_{0}$ is in the middle of the range considered by Szymanska and Littlewood ${ }^{12}$ for this geometry, and is close to the value reported by Butov et al. ${ }^{13}$ Using a dielectric constant appropriate to GaAs of 13.2 we find $a_{B}^{*}=156 \AA$ and $\mathcal{R}^{*}=3.5 \mathrm{meV} . E_{b}$ is sensitive to the value of $d$, and therefore we fix its value such that we reproduce the large-field exciton binding energy of $4 \mathrm{meV}$ $\left(1.14 \mathcal{R}^{*}\right)$ calculated for this structure by Szymanska and Littlewood, ${ }^{12}$ who used a realistic description of the finite well widths and depths. Using Eq. (3) we find $d=0.64 a_{B}^{*}(100 \AA)$, which is a very reasonable value as it lies between the experimental barrier width of $40 \AA$ and the distance between the centers of the wells of $120 \AA .{ }^{1}$ Substituting $d=0.64 a_{B}^{*}$ into Eq. (6) and using the parameters for $\sigma=2$ we obtain $E_{b}=8.2 \times 10^{-5} \mathcal{R}^{*}\left(2.9 \times 10^{-4} \mathrm{meV}\right)$. This model predicts an extremely small biexciton binding energy, which is unlikely to lead to measurable effects.

In conclusion, we have calculated the energies of excitons and biexcitons in ideal two-dimensional bilayer systems within the effective-mass approximation with isotropic electron and hole masses. The exciton binding energy decays as the inverse of the layer separation, while the biexciton binding energy with respect to dissociation into two separate excitons decays exponentially. This model indicates that biexciton formation, which would hinder Bose-Einstein condensation of excitons, is effectively suppressed in the geometry used by Butov et al. ${ }^{1}$

We thank Peter Littlewood for useful discussions. N.D.D. and R.J.N. acknowledge financial support from the Engineering and Physical Sciences Research Council (EPSRC), U.K.
${ }^{1}$ L. V. Butov, A. C. Gossard, and D. S. Chemla, Nature (London) 418, 751 (2002).

${ }^{2}$ D. Snoke, S. Denev, Y. Liu, L. Pfeiffer, and K. West, Nature (London) 418, 754 (2002).

${ }^{3}$ L. V. Butov, L. S. Levitov, A. V. Mintsev, B. D. Simons, A. C. Gossard, and D. S. Chemla, Phys. Rev. Lett. 92, 117404 (2004).

${ }^{4}$ R. Rapaport, G. Chen, D. Snoke, S. H. Simon, L. Pfeiffer, K. West, Y. Liu, and S. Denev, Phys. Rev. Lett. 92, 117405 (2004).

${ }^{5}$ W. M. C. Foulkes, L. Mitas, R. J. Needs, and G. Rajagopal, Rev. Mod. Phys. 73, 33 (2001).

${ }^{6}$ T. Kato, Commun. Pure Appl. Math. 10, 151 (1957).

${ }^{7}$ M. A. Lee, P. Vashishta, and R. K. Kalia, Phys. Rev. Lett. 51, 2422 (1983).

${ }^{8}$ C. J. Umrigar, K. G. Wilson, and J. W. Wilkins, Phys. Rev. Lett.
60, 1719 (1988)

${ }^{9}$ P. R. C. Kent, R. J. Needs, and G. Rajagopal, Phys. Rev. B 59, 12344 (1999).

${ }^{10}$ R. J. Needs, M. D. Towler, N. D. Drummond, P. R. C. Kent, and A. Williamson, CASINO version 1.6 User Manual (University of Cambridge, Cambridge, 2002).

${ }^{11}$ D. Bressanini, M. Mella, and G. Morosi, Phys. Rev. A 57, 4956 (1998); K. Varga, J. Usukara, and Y. Suzuki, Phys. Rev. Lett. 80, 1876 (1998).

${ }^{12}$ M. H. Szymanska and P. B. Littlewood, Phys. Rev. B 67, 193305 (2003).

${ }^{13}$ L. V. Butov, A. V. Mintsev, Yu. E. Lozovik, K. L. Campman, and A. C. Gossard, Phys. Rev. B 62, 1548 (2000). 